msh-mss Mathématiques et sciences humaines

139 | Automne 1997

Varia

\title{
J.-L Desalles, "L'ordinateur génétique", Paris,
} Hermès, 1996

J.-L Desalles, "L'ordinateur génétique", Paris, Hermès, 1996

Olivier Hudry

\section{(2) OpenEdition}

1 Journals

Édition électronique

URL : http://journals.openedition.org/msh/2814

DOI : $10.4000 / \mathrm{msh} .2814$

ISSN : 1950-6821

Éditeur

Centre d'analyse et de mathématique sociales de l'EHESS

Édition imprimée

Date de publication : 1 septembre 1997

ISSN : 0987-6936

Référence électronique

Olivier Hudry, « J.-L Desalles, "L'ordinateur génétique", Paris, Hermès, 1996 », Mathématiques et sciences humaines [En ligne], 139 | Automne 1997, mis en ligne le 10 février 2006, consulté le 25 septembre 2020. URL : http://journals.openedition.org/msh/2814 ; DOI : https://doi.org/10.4000/msh 2814

(c) École des hautes études en sciences sociales 


\section{BIBLIOGRAPHIE}

DESSALLES, J.-L., L'ordinateur génétique, Paris, Hermès, 1996.

Les algorithmes génétiques sont nés au début des années soixante pour simuler l'évolution de systèmes vivants. Depuis, ils ont été développés dans divers domaines, dont l'optimisation combinatoire où ils apparaissent comme une méthode générale approchée (ce qu'on appelle aussi une "méta-heuristique"), car leur principe est suffisamment général pour qu'ils puissent être adaptés à un grand nombre de problèmes fort différents dans leurs modélisations et leurs résolutions. Ils s'inspirent du vivant, et plus précisément de la génétique, tant pour leur nom que pour leur conception : les ingrédients qu'ils comportent sont la sélection, le croisement, et la mutation, termes qui évoquent le domaine où les algorithmes génétiques puisent leurs racines. Contrairement aux méthodes traditionnelles, les algorithmes génétiques ne considèrent pas une seule "solution" du problème à résoudre, mais une population de telles configurations dont on espère qu'elles pourront conduire à des solutions satisfaisantes, voire optimales, à l'aide d'une évolution régie par les opérateurs mentionnés plus haut.

Le livre de Jean-Louis Dessalles intitulé L'ordinateur génétique, publié aux éditions Hermès (collection Systèmes complexes) est consacré aux algorithmes génétiques, mais pas seulement sous l'angle purement technique (même si cet aspect est largement développé), se démarquant ainsi des manuels traditionnels. Le premier chapitre ("Programme génétique et programme informatique") constitue une introduction au domaine, entre autres à partir d'un exemple qui sera filé tout au long de l'ouvrage : celui d'un labyrinthe dont on cherche à sortir. Les trois chapitres suivants ("Laisser faire la nature", "Le schéma égoïste" et "La puissance du croisement génétique") sont consacrés à des rappels sur la théorie de l'évolution, abordée bien sûr dans une perspective algorithmique. Le chapitre 5 ("Le fonctionnement d'un algorithme génétique") revient sur les ingrédients des algorithmes génétiques et les détaille en mettant en évidence les propriétés qu'il est souhaitable qu'ils possèdent. Le chapitre 6 aborde la question du "parallélisme implicite" qui s'exerce au niveau des "schémas" : les schémas (chromosomes partiellement spécifiés) jouent un rôle majeur dans l'efficacité des algorithmes génétiques et il est souvent avancé que celle-ci provient de l'aptitude des algorithmes génétiques à traiter simultanément les bons schémas plutôt que les bons individus de la population (ceux-ci n'apparaissant plus que comme des "paquets de schémas") ; c'est de ces thèmes qu'il est question dans ce chapitre. Revenant au parallèle développé entre la génétique et les algorithmes du même nom, l'auteur s'interroge dans le chapitre 7 ("Evolution et optimalité") pour savoir ce qu'apportent les algorithmes génétiques et ce que l'on est en droit d'attendre d'eux, ici encore en s'appuyant sur ce que l'on peut observer dans la nature. Le chapitre 8 ("Les algorithmes biomimétiques") conclut le livre mais ne clôt pas le sujet ; au contraire, y sont abordées diverses questions qui élargissent le débat, ouvrant la voie à un large domaine dont l'objectif est de s'inspirer du monde vivant et d'en adapter les principes à une autre discipline.

On le constate donc, le propos de l'auteur n'était pas d'écrire un traité exhaustif sur la mise en œuvre et les applications des algorithmes génétiques; ce que confirme l'avant-propos : "Ce livre a pour objet d'introduire les concepts [c'est l'auteur qui souligne] fondamentaux sur lesquels reposent les algorithmes génétiques. Ces concepts proviennent de la génétique naturelle et de la théorie de l'évolution des êtres vivants. Il ne s'agit pas ici d'envisager les innombrables applications des algorithmes génétiques (...). Il s'agit plutôt de comprendre la raison d'être de chacune des composantes d'un algorithme génétique (...) et leur rôle dans la découverte de bonnes solutions". De ce fait, ce livre ne se limite pas à une présentation technique des algorithmes génétiques, même si on y trouve une description complète et détaillée du fonctionnement de ces méthodes, permettant ainsi au lecteur intéressé de les mettre en œuvre. C'est aussi ce qui explique que le livre ne s'attarde guère sur les applications industrielles ou 
plus académiques auxquelles ont donné lieu ces méthodes : c'est qu'il s'agit bien plutôt d'une réflexion sur les liens entre ces méthodes d'optimisation et les principes de l'évolution que l'on peut observer dans la nature. Et cette réflexion, fouillée et convaincante, agit à double sens : si la génétique nous donne des modèles sur l'évolution qui peuvent être féconds en algorithmique, réciproquement J.-L. Dessalles montre, par exemple en considérant les équilibres ponctués, la non-hérédité des caractères acquis ou encore les propriétés du codage génétique (artificiel ou naturel), qu'une maîtrise des mécanismes et du réglage des paramètres des algorithmes génétiques contribue à une meilleure compréhension du rôle de ces mêmes composantes du monde vivant.

Ceci répond à la question de savoir qui peut lire cet ouvrage avec profit : les algorithmiciens bien sûr, qui trouveront là une description des algorithmes génétiques répondant de manière totalement satisfaisante à leurs besoins ; mais aussi les chercheurs non nécessairement férus d'informatique qui tentent de mieux comprendre les mécanismes de la nature. Ce n'est pas là la moindre originalité de ce livre que d'offrir ainsi une lecture multiple, nourrie à la fois de considérations liées aux théories de l'évolution (en particulier la théorie du "gène égoïste" de R. Dawkins) et d'expériences algorithmiques, la plupart développées par l'auteur (c'est le cas notamment du problème du labyrinthe qui illustre les différents chapitres). On pourrait ajouter d'autres qualités à l'originalité de la démarche, notamment la clarté de la présentation et la pertinence de l'argumentation... Il en résulte un livre profond et stimulant qui, au-delà des aspects techniques des algorithmes génétiques, invite à une réflexion sur les fondements mêmes de ces méthodes et sur ce que le "biomimétisme" peut nous apprendre en retour sur la nature de l'être vivant et du monde dans lequel il évolue. 